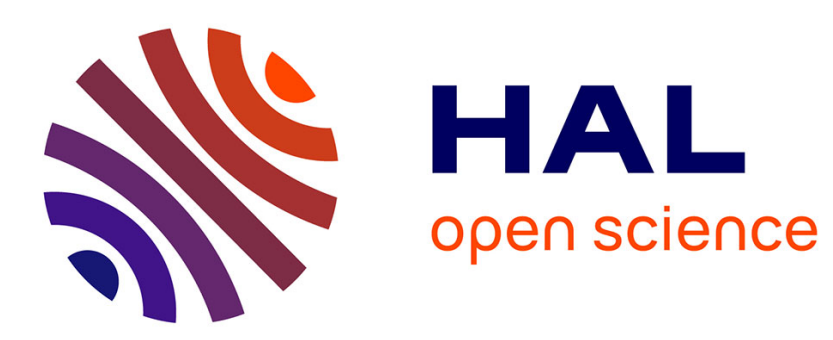

\title{
Normal oscillations of a string with concentrated masses on non-linearly elastic supports
}

\author{
Elena Vedenova, Leonid Manevich, Valery Pilipchuk
}

\section{To cite this version:}

Elena Vedenova, Leonid Manevich, Valery Pilipchuk. Normal oscillations of a string with concentrated masses on non-linearly elastic supports. Journal of Applied Mathematics and Mechanics, 1985, 49 (2), pp.153-159. 10.1016/0021-8928(85)90095-4 . hal-01510835

\section{HAL Id: hal-01510835 \\ https://hal.science/hal-01510835}

Submitted on 20 Apr 2017

HAL is a multi-disciplinary open access archive for the deposit and dissemination of scientific research documents, whether they are published or not. The documents may come from teaching and research institutions in France or abroad, or from public or private research centers.
L'archive ouverte pluridisciplinaire HAL, est destinée au dépôt et à la diffusion de documents scientifiques de niveau recherche, publiés ou non, émanant des établissements d'enseignement et de recherche français ou étrangers, des laboratoires publics ou privés.

\section{(c)(1)}

Distributed under a Creative Commons Attribution| 4.0 International License 


\title{
NORMAL OSCILLATIONS OF A STRING WITH CONCENTRATED MASSES ON NON-LINEARLY ELASTIC SUPPORTS
}

\author{
E.G. VEDENOVA, L.I. MANEVICH, and V.N. PILIPCHUK
}

\begin{abstract}
The problem of constructing and classifying normal oscillations of a string with concentrated masses on non-linearly elastic supports is considered (special limit cases are linear and vibro-impact systems). It is shown that in the limit of intensive impact action, the non-linear system has supplementary properties of symetry which enables this problem to be solved effectively. On the other hand, the nomal oscillations of a vibro-impact system can be used as the generating solutions for dynamic calculations of essentially non-linear systems that are close to them. The connection between localizec normal oscillations and solutions of the soliton type are discussed.
\end{abstract}

The existence of nomal oscillations as special particular solutions of linear conservative system is due to the properties of symmetry inherent in it that can be partly retained in the non-linear case $/ 1,2 /$. The possibility of constructing in some strongly non-linear system synchronous motions that have a number of properties of normal linear oscillations /3, $4 /$ is related to this property. The nomal "principal" oscillations have already been considered by Lyapunov $/ 5 /$. Due to these properties a multidimensional non-linear system in the normal oscillation mode behaves as a jon-linear oscillator.

1. Consider the transverse oscillations of a regular chain of masses connected to each other by a weightless string, and interacting with non-linearly elastic supports (Fig.i) which in the limit become rigid imiters with some gap $2 e$.

The reaction of the $j$-th support is

$$
q_{j}=a\left(u_{j}, e\right)^{2 n-1}
$$

where $u_{j}$ is the deflection of the string in the respective cross section, $n$ is an integer, and $a$ is the stiffness parameter.

Among the various type of motions of non-linear systems are those of the simplest mode, such as normal oscillations. When $n=1$ (a string with concentrated masses on linearly elastic supports) we have $N$ nomal oscillations; the spectrum of respective natural frequencies is discrete and limited.

When $n>1$ we distinguish the cases of strong and weak connection along the string. In the first case the non-linearity is small and the system belongs to the Lyapunov class of systems $15 /$. We thus have the problem of constructing non-linear nomal oscillations which, as the amplitude decreases, become normal oscillations of a normalized system. This simple case is not considered further. 


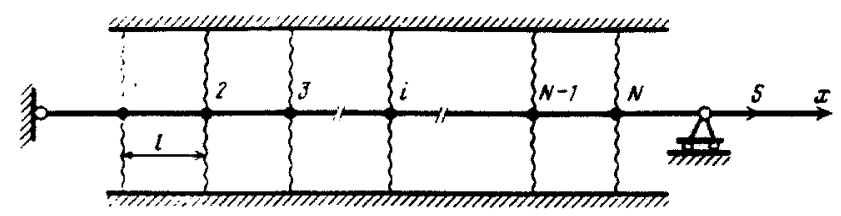

Fig.1

In the second case, if we neglect the connection along the chain, we obtain a system of non-interacting homogeneous oscillators (the potential energy of each oscillator is a homogeneous function of the displacement). Exact solutions of that system are, however, difficult to use as the first approximation in any scheme of the theory of perturbation, since they are expressed in terms of special functions. At the same time, the properties of the oscillation process, including also the mode of normal oscillations, essentially depends now on the index $n$. When $n \sim 1$ (e.g. $n=2,3$ ) normal oscillations are still close to harmonic (in time) and, consequently they can be defined in terms of the quasiharmonic approximation in the standara scheme of the method of averaging. If, however, $n \gg 1$ the system behaviour proves to be closer to the other limit case $(n \rightarrow \infty)$ that corresponds to the characteristic of the elastic medium shown in Fig.2, i.e. to an impact interaction. The investigation of normal oscillations of systems of the class of the situation considered in these circunstances which are the least favourable from the point of view of the quasiharmonic approach, is the basic content of this paper.

2. Let us first consider a string with two unit concentrated masses in the limit case of impact interaction, when the non-linearly elastic supports become rigid limiters. In that case the equations of motion are

$$
\begin{aligned}
& \frac{d^{2} u_{1}}{d t^{2}}-\because\left(2 u_{1}-u_{2}\right)-q\left(u_{1}, \frac{d u_{1}}{d t}\right)=0 \\
& \frac{d u_{2}}{c t}-\because\left(2 u_{2}-u_{1} \mid-q\left(u_{2}, \frac{d u_{2}}{d t}\right)=0\right. \\
& \left|u_{1}\right|<e \cdot\left|u_{2}\right| \cdots e: \quad \eta=S l
\end{aligned}
$$

where $g$ is the stress impact interaction which cuts-in when $\left|u_{j}\right|=e(j=1,2)$, $S$ is the tension of spring, and $l$ is the distance between the supports (masses).

Wher $i=U$ (there is no connection) both masses move independently of each other, undergoing only absolutely elastic impact against the elastic supports. The respective coordinates $u_{i}(j-1,2)$ are determined as follows:

$$
\left.u_{j}=\epsilon\right](4): \frac{c r}{6 r}=\frac{1}{2} \frac{\pi !}{e}
$$

where $I l(4)$ is a saw-tooth function of period 27 and amplitude $1,4 j$ is phase of the $j$-th mass, and $i$, is the absolute velocity, constant in time.

Passing to the general case $(\gamma \neq(1)$. we shall consider (2.2) as the change of variables. Such rough transformations were first utilized in $/ 6 /$. The equations of motion then reduce to the form.

$$
\begin{aligned}
& \frac{d^{2} \tau_{1}}{a t_{2}}-\gamma \frac{T^{2}}{4} \Pi\left(\psi_{3}\right)\left[2 \Pi\left(\varphi_{1}\right)-\Pi\left(\psi_{2}\right)\right]=0 \\
& \frac{d^{2} \psi_{2}}{d t^{2}}-\gamma \frac{\bar{z}^{2}}{4} \Pi\left(\varphi_{2}\right)\left[2 \Pi\left(\psi_{2}\right)-\Pi\left(\psi_{1}\right)\right]=0 .
\end{aligned}
$$

The syster (2.3), uniike (2.1) does not contain functions with discontinuities of the second kind.

Relations (2.3) may be considered as the equations of motion of unit mass in the $\left(\varphi_{1} . \psi_{2}\right)$ plane in a periodic potential field of force; when $\gamma=0$ we have inertial motion.

A set of equipotential lines is shown in Fig.3, where the trajectory in the plane $\left(\varphi_{1} . \varphi_{2}\right)$ is mapped, using (2.2) into the configurational space of the initial system $\left(u_{1}\right.$, $\left.u_{2}\right)$. That image "turns" the plane $\left(\varphi_{1}, \varphi_{2}\right)$ along the lines

$$
\varphi_{1}=\frac{\pi}{2} \div j x ; \quad \varphi_{2}=\frac{\pi}{2}-j \pi \quad(j= \pm 1,=2, \ldots)
$$

into a square $K:\left\{-\pi 2 \leqslant \varphi_{i} \leqslant \pi 2, i=1,2\right\}$ with subsequent stretching in the directions $\psi_{1}, \varphi_{2}$ $e$ times. For fairly small energies the system moves without inpacts so that the trajectory 


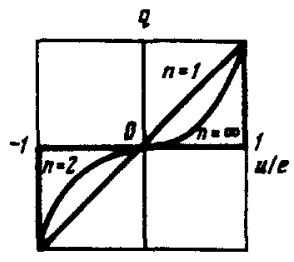

Fig. 2

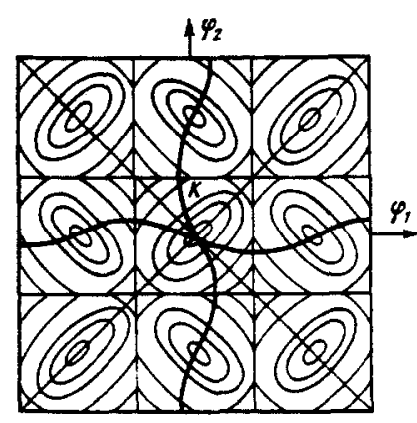

Fig. 3

in the plane $\left(\psi_{1}, \varphi_{2}\right)$ remains inside the square $K$. Passing into a new element of the plane corresponds to an impact on the limitex.

Note that the pattern in square $K$ (the equipotential curves of a linear system) has an axis of rotational symmetry of second order, and the gap (Fig.3) obtained by multiple mirror reflection of that pattern (the motive) relative to the straight lines (2.4) has a fourthorder axis of symetry. This is related to the substantial difference of the dynamic properties of the linear model (without impact) and modes of motion with impacts against a limiter. Thus, in the first instance we have two nomal oscillations, i.e. the natural oscillations of a linear system with two degrees of freedom. With impacts, there are already four (Fig.3), since besides a symmetric (inphase) and skew-symmetric (antiphase) forms, two supplenentary spatially localized forms appear, which at the limit $\gamma \rightarrow 0$ become straight lines $\varphi_{1}=0, \varphi_{2}=0$ and correspond to one of the masses being at rest.

3. The part played by normal oscillations is primarily dependent on the possibility of their use as providers of solutions when equations of motion are asymptotically integrated. Because of this, we shall obtain the approximate (averaged) equations which defines the behaviour of a system about forms enumerated above. When constructing such approximate equations it is advisable to utilize the intrinsic small parameter of the problem that reflects the basic property of the impact mode, $i . e$. the relatively small magnitude of the coupling parameter

$$
\varepsilon^{2}=4 e^{2} \gamma\left(\pi^{2} v^{2}\right)
$$

Physically the constraint $\varepsilon^{2} \ll 1$ is obviously connected with the condition of realizing impacts. The smallness of the parameter $\varepsilon$ predetermines the availability in the system of "rapid" and "slow" times, which enables us to use the averaging method in this or that modification. Below, in each case we present only shortened equations that correspond to the first approximation. Classification of normal forms by the type of behaviour in the neighbourhoods of these forms is carried out on this basis.

Inphase form $\varphi_{1}=\varphi_{2}$. We set $\varphi_{1}=\tau+\bar{\varphi}_{1}\left(t_{1}\right), \varphi_{2}=\tau+\bar{\varphi}_{2}\left(t_{1}\right)$, where $\tau={ }^{1} \cdot{ }_{2} \pi e^{-1} t$ is the rapid and $t_{1}=\varepsilon \tau$ the slow time, respectively. The rapid time is, naturally, related to the rate of impact.

Carrying out the averaging in $(2,3)$ over the variable $\tau$, we obtain

$$
\frac{d^{2}\left(\bar{\varphi}_{1}-\bar{\varphi}_{2}\right)}{d t_{2}^{2}}=0, \quad \frac{d^{2}\left(\bar{\Phi}_{1}-\bar{\varphi}_{2}\right)}{d t_{1}^{2}}-2 \Phi\left(\bar{\varphi}_{1}-\bar{\varphi}_{2}\right)=0
$$

where $\Phi(\xi)$ is a 2.x-periodic function defined on the segment $[-\pi, \pi]$ by the formula

$$
\Phi(\xi)=\frac{\pi^{2}}{4}\left\langle\Pi^{\prime}(\tau) \Pi(\tau+\xi)\right\rangle_{\tau}=\left\{\begin{array}{l}
\xi(1-\xi / \pi), 0 \leqslant \xi \leqslant \pi \\
\xi(1+\xi / \pi),-\pi \leqslant \xi<0
\end{array}\right.
$$

The trivial solution of the averaged system corresponding to the inphase form is orbitally stable in the "small". In this sense we refer to the inphase form as of the "stable" type.

Antiphase form $\varphi_{1}=-\varphi_{2}$. Let $\varphi_{1}=\tau+\bar{\varphi}_{1}\left(t_{1}\right), \bar{\varphi}_{2}=-\tau+\bar{\varphi}_{2}\left(t_{1}\right)$. In this case the equations relative to $\bar{\varphi}_{1}, \bar{\varphi}_{2}$ may be reduced to the form

$$
\frac{d_{2}\left(\bar{\varphi}_{1}-\bar{\Phi}_{2}\right)}{d i_{1}{ }^{2}}=0 ; \frac{d^{2}\left(\bar{\Phi}_{1}+\bar{\varphi}_{2}\right)}{d t_{1}^{2}}-2 \Phi\left(\bar{\varphi}_{1}+\bar{\varphi}_{2}\right)=0
$$

The trivial solution of the averaged system corresponds to the antiphase form and is orbitaliy unstable. Hence we regard the antiphase form as being of the "unstable" type. 
Localized forms. Finally, let us consider the neighbourhood of the straight line $q_{2}=0$. As previously noted, that straight line is a nomal form only when $y=0$. However, when $\gamma \neq 0$, the curvilinear form is "on the average" directed along the straight line $\varphi_{2}=0$ (Fig.3). Let us assume that the trajectory in the course of motion remains in the band $-\pi / 2<\varphi_{2}<\pi / 2$. Eq. (2.3) then takes the form

$$
\begin{aligned}
& \frac{d^{2} \varphi_{1}}{d \tau^{2}}-\frac{\varepsilon^{2} \pi^{2}}{4} \Pi\left(\varphi_{1}\right) \Pi^{\prime}\left(\varphi_{1}\right)=\frac{\varepsilon^{2} \cdot \pi}{4} \Pi^{\prime}\left(\varphi_{1}\right) \psi_{2} \\
& \frac{d^{2} \varphi_{2}^{2}}{d \tau^{2}}-\varepsilon^{2} \Psi_{2}=\frac{\varepsilon^{2} \cdot \pi}{4} \Pi\left(\varphi_{1}\right) \\
& \varepsilon^{2}=\frac{8 e^{2} \eta}{\pi^{2} l_{1}{ }^{2}} \ll 1, \quad \tau=\frac{\pi v_{1}}{2 e} t
\end{aligned}
$$

Setting $\varphi_{1}=\tau+\bar{\varphi}_{1}\left(t_{1}\right)$ and averaging (3.1) over $\tau$, we obtain the equations

$$
\frac{d^{2} \bar{\tau}_{1}}{d t_{1}^{2}}=0, \quad \frac{d^{2} \bar{q}_{2}}{d t_{1}^{3}}+\bar{\Psi}_{2}=0
$$

which indicate orbital stability of the trajectory $\varphi_{2}=0$ relative to the perturbations

$$
\bar{\varphi}_{2}, \frac{d \bar{\varphi}_{2}}{d t_{1}} \text {. }
$$

This means that a reasonably large energy imparted in the initial instant to one of the oscillators is subsequently maintained by it. The localized form belongs in the sense indicated above to the "stable" type. A similar conclusion also holds for the second localized form.

4. A model with two degrees of freedom reveals an important feature that is inherent in all discrete systems of the class considered here, i.e. the existence of a form of localized oscillations. Other types of elenentary solutions that do not appear in linear systems emerge only when $N \geqslant 3$, as will be shown later. In this connection, we will now analyse a discrete model with three degrees of freedom.

In that case the equations that define the phases $\varphi_{i}(i=1,2,3)$ have the form

$$
\frac{d: \Phi_{2}}{d t^{2}}-\frac{\Pi^{2}}{4} \Pi^{\prime}\left(\varphi_{i}\right)\left[\Pi\left(\varphi_{i-1}\right)-2 \Pi\left(\varphi_{i}\right)+\Pi\left(\varphi_{i+1}\right)\right]=0
$$

where $\varphi_{0}=\varphi_{3} \equiv 0$. Introducing the dimensioniess parameter of time $\tau-1 / z^{\pi} e^{-1} t$, we write this system in the form

$$
\begin{aligned}
& \frac{d^{2} \Psi_{1}}{d \tau^{2}}-\gamma \frac{e^{2}}{v^{2}} \Pi^{\prime}\left(\psi_{1}\right)\left[2 \Pi\left(\psi_{1}\right)-\Pi\left(\psi_{2}\right)\right]=0 \\
& \frac{d^{2} \Psi_{2}}{d \tau^{2}}-\gamma \frac{e^{2}}{v^{2}} \Pi^{\prime}\left(\varphi_{3}\right)\left[-\Pi\left(q_{1}\right)-2 \Pi\left(\varphi_{2}\right)-\Pi\left(q_{3}\right)\right]=0 \\
& \frac{d^{2} \Psi_{3}}{d \tau^{3}}-\gamma \frac{e^{2}}{v^{2}} \Pi^{\prime}\left(\varphi_{3}\right)\left[-\Pi\left(\psi_{2}\right)-2 \Pi\left(\varphi_{3}\right)\right]=0 .
\end{aligned}
$$

Equations (4.1) may be considered as the equation of motion of a unit mass in threedimensional space in a periodic potential field of force. The equipotential surfaces of this field form a spatial pattem obtained by multiple mirror reflection of a cubic element - $\pi, 2 \leqslant$ $4_{i} \leqslant \pi .2(i=1,2.3)$. Inside the element the field of force corresponds to the linear problen with three degrees of freedom, and ahs three axes of symmetry of a triaxial ellipsoid. The field in the whole space of $\left(4_{1} .4_{2} .4_{3}\right)$ has 13 axes of symmetry of the cubic lattice, and hence, unlike the linear problem, 13 normal oscillations are possible here and not three. However the relations shown in Fig.4, comprise all essentially different cases (the remaining forms are easily reestablished on considerations of symmetry). Here $t_{1}=\varepsilon \tau ; \varepsilon^{2}=4 \gamma e^{2} /\left(\pi^{2} \imath^{2}\right)$. It is assumed in cases $1,2,5$ that $-\pi 2<\varphi_{i}<\pi-(i=1,2,3)$. The masses shown in Fig.4 as fixed, are at rest only in the "mean", executing high-frequency oscillations with small amplitudes.

These results show the existence of localized forms of oscillations of the "stable" type for systems with two degrees of freedom also (solutions 1, 2 and a similar form with localization on the third mass). At the same time a new effect appears in the system with three degrees of freedom: the possibility of cooperative motion of two adjacent masses (of the stable type) when there are connections undeformed on the average (solution 3 ). As in Sect. 3 , the in-phase form (solution 6) is of the stable type (denoted in Fig.4 by the plus sign), and the antiphase form (solution 7) of the unstable type (denoted by the minus sign). Other forms of motion in which displacements of two adjacent masses have different signs (solutions 4, 8), and, also, the form in which the fixed mass (on the average) separates two deformed connections (solution 2) also belong to the unstable type.

These results (Fig.4) enable us to represent the possible forms of normal oscillations for $N>3$ also. 


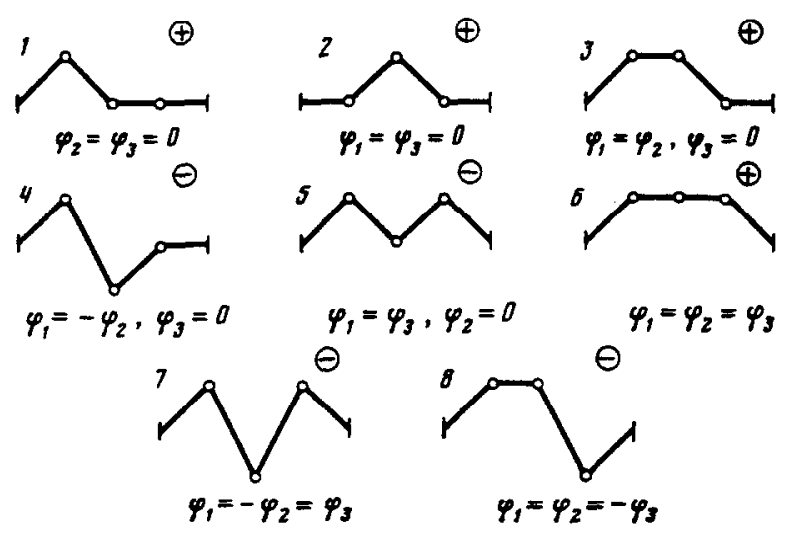

Fig. 4

5. We shall now show how the results related to the limit case $n \rightarrow \infty$ can be used to construct oscillations when $n \gg 1$.

As an example, we shall consider the system with two degrees of freedom

$$
\begin{aligned}
& \frac{d^{2} u_{1}}{d t^{2}}+\gamma\left(2 u_{1}-u_{2}\right)+u_{1}{ }^{k}=0 \\
& \frac{d^{2} u_{3}}{d t^{2}}-\gamma\left(2 u_{2}-u_{1}\right)-u_{2}{ }^{k}=0 \quad(k=2 n-1)
\end{aligned}
$$

We shall seek the normal form of oscillations that corresponds to the localized form of the limit system $(k \rightarrow \infty)$.

We set

$$
\begin{aligned}
& u_{1}=u_{0}+v^{-2} U\left(u_{0}\right), \quad u_{2}=v^{-2} V\left(u_{0}\right) \\
& u_{0}=A \Pi(\varphi), \quad \varphi=v t / A
\end{aligned}
$$

(the period of the function $\Pi$ is provisionally taken as equal to 4). Substituting (5.2) into (5.1), we obtain the following system of equations in $U\left(u_{0}\right), V\left(u_{0}\right)$ :

$$
\begin{aligned}
& l^{\cdots} \div\left\{A^{-1}\left(l^{2}-L^{\prime}\right) \Pi^{\prime \prime}\right\}+2 \gamma u_{0}+\gamma l^{-2}(2 U-V) \div \\
& \left(u_{0}+v^{-2} U\right)=0
\end{aligned}
$$

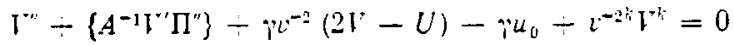

Setting

$$
L^{\prime \prime}{ }_{u=t}=-\left.i^{* 2} \cdot \quad V^{\prime \prime}\right|_{u_{r}=A}=0
$$

we eliminate the terms in braces which correspond to a periodic system of pulses ( $\delta$ functions) in the variable $\varphi$.

The first of conditions (5.4) is satisfied by the appropriate selection of the amplitude $A$, and the second by selecting the constants of integration in solving the equation in $V$. Writing $U\left(u_{0}\right), V\left(u_{0}\right)$ in the form

$$
\tau^{*}=U_{1}+v^{-2} U_{2}+\ldots, V=v_{1}+v^{-2} V_{2}+\ldots
$$

we obtain from (4.3) to a first approximation

From this we obtain

$$
\zeta_{1}^{\prime \prime}-2 u_{0}-u_{0}{ }^{i}=0 . \quad V^{\prime \prime}-\gamma u_{0}=0
$$

$$
L_{1}=-\gamma \frac{u_{0}^{3}}{3}-\frac{u_{0}^{k+2}}{(k+2)(k-1)}, \quad V_{1}=\gamma\left(\frac{u_{0}^{3}}{6}-\frac{A^{2} u_{0}}{2}\right)
$$

The equation for $A$ has the form

$$
r^{2} A^{2}-A^{k+1} /(k-1)=r^{2}
$$

Thus the solution in this approximation has the form

$$
\begin{aligned}
& U=u_{0}-\frac{\gamma}{3 v^{2}} u_{0}^{3}-\frac{u_{0}^{k+2}}{v^{8}(k+2)(k+1)} \\
& \mathrm{V}=\frac{\gamma}{l^{2}}\left(\frac{u_{0}^{3}}{6}-\frac{A^{2} u_{0}}{2}\right), \quad A \approx\left[(k+1) v^{2}\right]^{1(k+1)}
\end{aligned}
$$


We can similarly construct the solutions in subsequent approximations, with the amplitude A represented by the expansion

$$
A=A_{1}+v^{-2} A_{2}+\cdots
$$

6. Since the classification of normal forms was carried out using averaged equations, a numerical integration of the initial system with the characteristic $q_{j}=u_{j}{ }^{21}$ was carried out, corresponding in practice to the impact process with initial conditions specified in the "neighbourhood" of "normal" forms of the "stable" and "unstable" types.

Below, we present the results obtained for a model with two degrees of freedom for $v=$ $0.5 ; a=1 ; e=1 \quad$ (Fig. 5).
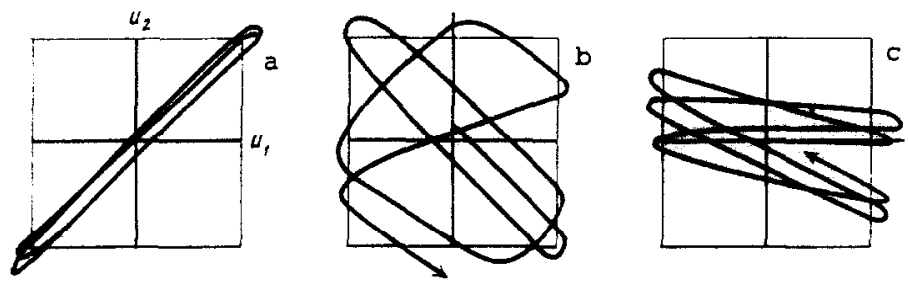

Fig. 5

When the normal form relates to the stable type, with initial conditions somewhat different from the "exact", the trajectory remains close to the form. This accurs for the inphase (Fig.5,a) and localized (Fig.5,c) forms. If, however, the forms belong to the unstable type (Fig.5,b, the antiphase form), a rapid "drift" of the trajectory from the form is observed.

7. Let us consider the connnectior between nomal oscillations and the soliton type solutions for an infinite chain.

If in this systen: $N \rightarrow \infty, e=1$, the equations of motion in a finite-difference approximations of elastic forces in the string have the form

$$
\begin{gathered}
d^{2} u_{j} / d t^{2}+\gamma\left(2 u_{j}-u_{:-1}-u_{j-1}\right)+a u_{j}^{2 n-1}=0 \\
j=0, \pm 1, \pm 2, \ldots
\end{gathered}
$$

This system has an exact particular solution that corresponds to the antiphase normal form

$$
u_{j}=(-1)^{j} U(t)
$$

where the function $U^{*}(t)$ satisfies the non-linear differential equation

$$
d^{2} U i d t^{2}+4 \gamma U+a U^{2-1}=0 .
$$

A solution of the soliton type can be sought in the class of weakly modulated standing waves

$$
u_{j}=(-1)^{j} U_{j}(t)
$$

assuming for every $t$ a dependence of the functions $U_{j}(t)$ on the index $j$ that is smooth compared with $u_{j}(t)$. After substituting (7.3) into (7.1) and simple transformations, we obtain

$$
\begin{aligned}
& d^{2} U_{j} d t^{2} \div \gamma\left(U_{j+1}+U_{j-1}-2 U_{j}\right)+4 \gamma U_{j}+a U_{j}^{2 n-1}=0 \\
& j=0, \pm 1, \pm 2, \ldots
\end{aligned}
$$

By virtue of the smooth dependence on $j$, the set of functions $U_{j}(t)$ can be approximated by one function of two variables $(U(x, t)$ and the finite difference operator in $(7.4)$ by the second partial derivative with respect to the space coordinate. We then have a single differential equation in partial derivatives with respect to $U(x, t)$

$$
\partial^{2} U / \partial t^{2}+\gamma l^{2} \partial^{2} U / \partial x^{2}+4 \gamma U+a U^{2 n-1}=0
$$

which corresponds to the use of continuous approximation for system (7.4). This non-linear equation has a localized solution of the soliton type with the provisional frequency $w$, that

exceeds the maximum natural oscillations of system (7.1) after linearization $\omega_{\max }=2 \sqrt{\gamma}$. If $\varepsilon^{2}=\omega^{2}-\omega_{\max }^{2} \ll 1$, then in the first order with respect to $\varepsilon$ the soliton solution may be expressed in explicit form. For instance, when $n=2 / 7-9 /$ 


$$
U=4 \varepsilon \sin \omega t \operatorname{sech} \frac{\varepsilon}{l \sqrt{\gamma}} x
$$

so that

$$
u_{j}=(-1)^{j} U\left(x_{j}, t\right)
$$

Solutions of the soliton type may be similarly obtained when $n>2$.

In the region of applicability of the continuous approximation $(7.5)$, when $\varepsilon \ll 1$ and the function $U(x, t)$ is in fact smoothly varying along the coordinate $x$, its localization is weakly expressed and the amplitude can be arbitrarily small. The linear and non-linear terms of system (7.5) are of the same order in $\varepsilon$.

As $E$ increases the amplitude increases, and the tendency to a pronounced localization becomes apparent. A soliton-type solution is virtually indistinguishable from a localized normal form. The continuous approximation and, consequently, the analytic representation (7.6) and (7.7) is no longer applicable here. In that case, the analysis carried out above in terms of normal oscillations is the most reasonable.

The analytic formulae of the form (7.6) and (7.7) and the localized normal forms investigated in this paper relate to one type of solution but correspond to different regions of amplitude variation.

\section{REFERENCES}

1. MANEVICH L.I. and PINSKII M.A., On normal oscillations in non-linear systems with two degrees of freedom. Prikl. Mekhanika, Vol.8, No.9, 1972.

2. MANEVICH L.I. and PINSKII M.A., On the use of symetry when calculating non-linear oscillations. Izv. AN SSSR, MTT, No.2, 1972.

3. ROSENBERG R.M., On the variation of systems with many degrees of freedom. In advances in Applied Mechanics. Vol.9, New York-London Acad. Press, 1966.

4. MANEVICH L.I. and MIKHIIN IU.V., On periodic solutions close to rectilinear vibration modes. PMM, Vol.36, No.6, 1972 .

5. MALKIN I.G., Some Problems of the Theory of Non-linear Oscillations. Moscow, Gostekhizdat. 1956.

6. ZHURAVLEV V.F., A method of analysing vibro-impact systems using special functions. Izv. AN SSSR. MTT, NO.2, 1976.

7. KOSEVICH A.M. and KOVALEV A.S., Selflocalization of oscillations in a one-dimensional anharmonic chain. ZHETF, Vol.67, No.5, 1974.

8. VEDENOVA E.G. and MANEVICH L.I., Periodic and localized waves in vibro-impact systems of regular structure. Mashinovedenie, No.4, 1981.

9. VEDENOVA E.G., Solitons in essentially non-linear system of impact type. Mashinovederie, No.1, 1983. 